\title{
The slow dielectric Debye relaxation of monoalcohols in confined geometries
}

\author{
Helén Jansson and Jan Swenson ${ }^{\mathrm{a})}$ \\ Department of Applied Physics, Chalmers University of Technology, SE-412 96 Göteborg, Sweden
}

(Received 23 November 2010; accepted 16 February 2011; published online 10 March 2011)

\begin{abstract}
Broadband dielectric relaxation measurements have been performed on monoalcohols confined in the quasi-two-dimensional space between clay platelets and the quasi-one-dimensional pores of approximately $10 \AA$ diameter in a molecular sieve. Interestingly, the results show that the slow Debye-like process is present even in these severe confinements, proving that structural models that are based on two-dimensional or three-dimensional cluster formations as the structural origin of the Debye-like process can be excluded. Rather, the insensitivity of its time-scale to confinements suggests that it is of local character and in some way related to the lifetime or breaking and reformation of hydrogen bonds. (C) 2011 American Institute of Physics. [doi:10.1063/1.3563630]
\end{abstract}

\section{INTRODUCTION}

Glass forming materials can be characterized by their dynamical behavior. The most studied, and probably also the most important, relaxation is the $\alpha$-relaxation that is directly coupled to the viscosity and the glass transition of the material. The main features of this structural relaxation are its non-Debye behavior and the often dramatic increase in relaxation time when approaching the glass transition. In addition to the $\alpha$-relaxation most glass forming liquids also exhibit faster secondary $\beta$-relaxations. These relaxations are of more local nature than the $\alpha$-relaxation, and are present even in the glassy state when the structural relaxation has frozen in. In addition to these commonly observed relaxations some other liquids, such as monoalcohols (see, e.g., Refs. 1-3), polyalcohols, ${ }^{4}$ monosaccharides,${ }^{5}$ and amorphous ibuprofen, ${ }^{6}$ display also a slower dielectric relaxation of Debye-type. The origin of this slow relaxation, which has only been observed in hydrogen bonded liquids, is not clear. It is neither established whether its origin is the same in all types of liquids where the process has been observed. In the case of monoalcohols the Debye-like process is considerably stronger than the structural $\alpha$-relaxation and therefore dominates the dielectric spectra, despite the fact that it cannot be readily observed with other techniques, such as, e.g., mechanical spectroscopy ${ }^{3}$ or differential scanning calorimetry. ${ }^{7}$ Many different models have been proposed in order to explain the structural origin of the dielectric Debye-like process in monoalcohols, and these are generally based on the formation of some kind of large hydrogen bonded structures, ${ }^{2,8,9}$ such as clusters, chains, or micelles. On the contrary, from molecular dynamics simulations of amorphous ibuprofen it was recently suggested that the Debye-like peak in this material is dominated by intramolecular cis-trans isomerism. ${ }^{10}$ Nevertheless, since the role of hydrogen bonds for the structure and dynamics of hydrogen bonded liquids is crucial to determine for understanding

\footnotetext{
a) Author to whom correspondence should be addressed. Electronic mail:
} jan.swenson@chalmers.se. biological and other water containing systems it is obviously important to establish what is causing this Debye-like process.

In this study we have filled different geometrical confinements with monoalcohols in order to elucidate how the restricted geometries affect the dielectric properties of the Debye-like process. Generally, it is difficult to predict whether the relaxation dynamics becomes faster or slower in confinements compared to bulk, particularly in the supercooled regime where both observations are common. The reason for this is that there is a competition between confinement effects, which tend to speed up the dynamics, and surface effects, which normally slows down the dynamics. Depending on the temperature, size of the cavity compared to the length scale of the cooperative molecular motions, and the nature of the surface interactions (e.g., hydrophilic or hydrophobic) the relaxation dynamics will either slow down or speed up. In order to discriminate between these two effects it is often necessary to study the structure and the dynamics of the liquids in well-defined model systems and/or to change the surface interactions by surface modifications. However, in our study the main aim is not to elucidate these types of confinement and surface effects in detail, but to restrict the number of possible structural origins of the Debye-like process. Neither we have searched for the exact mechanism behind it. The results show that the Debye-like process remains even in one-dimensional and two-dimensional geometries, where the liquids are severely restricted to about $1 \mathrm{~nm}$ in the other directions. Thus, by simple geometrical considerations we can exclude some of the structural models, such as cluster and micelle formations ${ }^{8}$ that have been proposed to explain its behavior. Still remaining are models that are based on chainlike one-dimensional structures, in analogy with the normal mode for polymers. ${ }^{2}$ However, since the relaxation time and the dielectric strength of the Debye-like process exhibit completely different alterations in confinements than the normal mode process, such an analogy seems unlikely as an explanation of the Debye-like process. Rather, our results suggest that no large structural formations are responsible for the Debye-like 
process, which more likely is of local character and related to the behavior of single hydrogen bonds.

\section{EXPERIMENTAL}

The samples investigated in this study were monoalcohols confined in a $\mathrm{Na}$-vermiculite clay and a molecular sieve, respectively. The monoalcohols 2-ethyl-1-hexanol (2E1H) (Fluka), 2-ethyl-1-butanol (2E1B) (Aldrich), and 2methyl-1-butanol (2M1B) (Aldrich) were studied in the $\mathrm{Na}-$ vermiculite clay, whereas only $2 \mathrm{E} 1 \mathrm{H}$ was confined in the molecular sieve, which is a commercial zeolite of type $13 \mathrm{X}$ from Sigma-Aldrich (catalogue No. M-3135). The molecular sieve has cages of $13 \AA$ diameter interconnected with channels of $8 \AA$ diameter. The network of these channels can be considered as infinitely extended, which means that the monoalcohol becomes confined in a quasi-one-dimensional geometry.

Vermiculite clays consist of a layer structure of thin and well-defined thickness. ${ }^{11}$ In this host material the monoalcohols are confined in the direction perpendicular to the clay platelets, but free to perform unrestricted movements in the two dimensions parallel to the clay platelets. In this study the $\mathrm{Na}-$ vermiculite clay was prepared from a vermiculite clay provided by Askania, Sweden, as described in Ref. 12. After that the clay and the molecular sieve had been dried at $150^{\circ} \mathrm{C}$ in a vacuum oven for at least $48 \mathrm{~h}$, they were submerged into each liquid for about 2 days. With this procedure the confining host materials were filled with the monoalcohols. In order to avoid contribution from the bulk liquid the surface of the materials was carefully dried before each measurement. The weigh and macroscopic thickness of the clay after it had been submerged into each of the monoalcohols were compared with the corresponding values directly after the vacuum drying. From this comparison we could conclude that the average interplatelet distance had increased to an interlayer thickness of the same order as the average pore diameter in the molecular sieve. From the number densities of the monoalcohols the diameters of the molecules have been calculated to 7.9, 7.3, and $7.0 \AA$ for $2 \mathrm{E} 1 \mathrm{H}, 2 \mathrm{E} 1 \mathrm{~B}$, and $2 \mathrm{M} 1 \mathrm{~B}$, respectively, assuming spherical geometry. Although the molecules are probably not fully spherical in shape, these values imply that only chain formations are possible, at least in the molecular sieve.

To be able to make a quantitative comparison with the corresponding bulk data we also performed dielectric measurements on bulk samples of the three monoalcohols $2 \mathrm{E} 1 \mathrm{H}$, 2E1B, and 2M1B. These monoalcohols do not easily crystallize in the supercooled regime and they are all known to display a pronounced Debye-like peak at lower frequencies than the $\alpha$-peak in the dielectric spectrum. ${ }^{13}$ To ensure that none of the samples lost any liquid during the measurements the weight of each sample was checked before and after each measurement.

The dielectric measurements were performed on a broadband dielectric spectrometer from Novocontrol, equipped with a Novocontrol Alfa-S high resolution dielectric analyzer. For each measurement the sample was placed between two stainless steel electrodes, which in the case of bulk liquids were separated by silica spacer fibers (Novocontrol) of

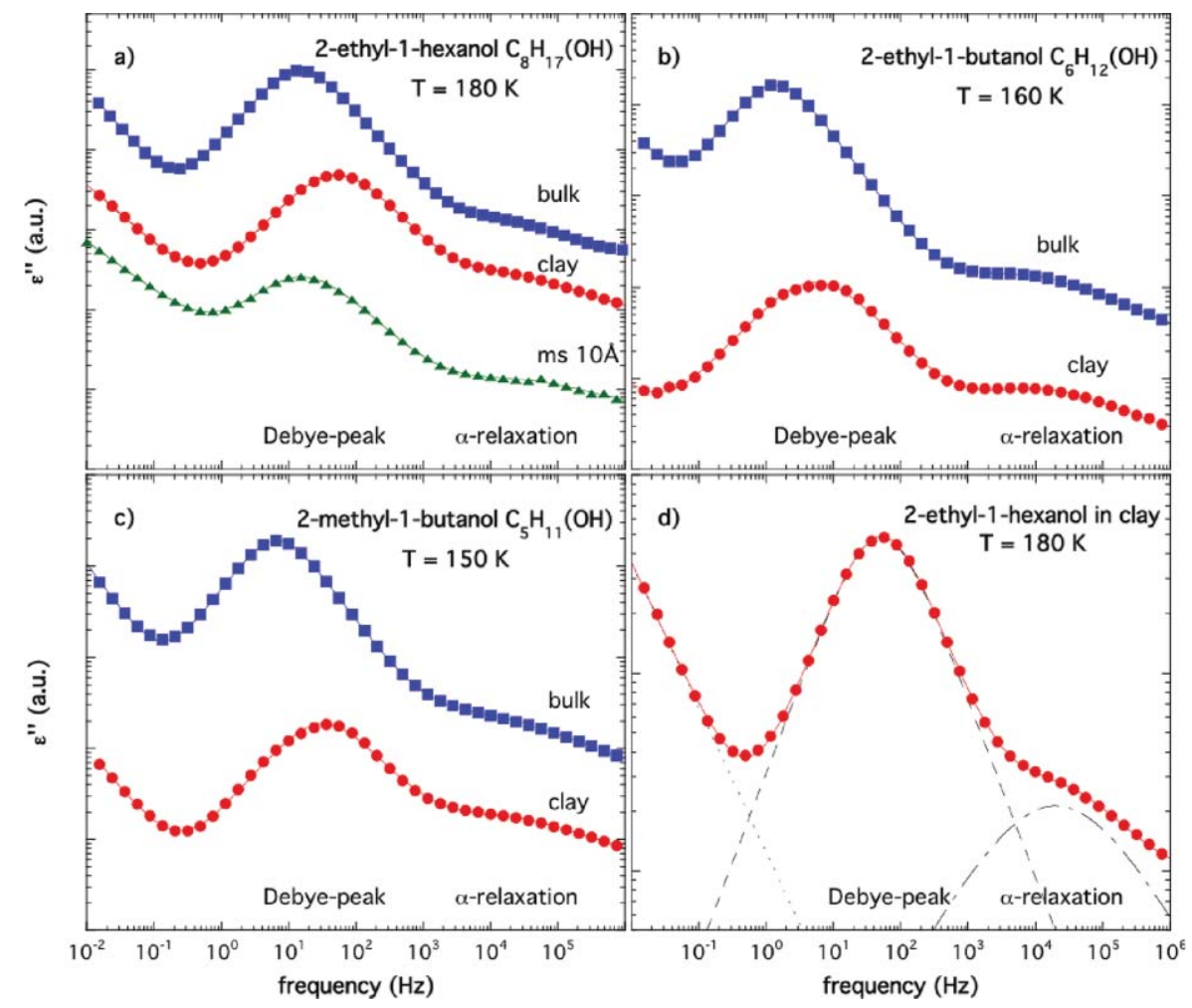

FIG. 1. Imaginary part of the permittivity of the monoalcohols (a) 2-ethyl-1-hexanol, (b) 2-ethyl-1-butanol, and (c) 2-methyl-1-butanol in confinement and bulk. The temperatures are given in the figures. In (d) it is shown how the data on 2-ethyl-1-hexanol in clay are curve fitted by a power law for the conductivity contribution (dotted line) and three Cole-Cole functions [Eq. (3)] for the Debye-like process (dashed line), the $\alpha$-relaxation (dashed-dotted line) and the $\beta$-relaxation (not shown). The total fit to the data is given by the solid line. 
diameter $0.1 \mathrm{~mm}$. The samples were first cooled down to a temperature well below its calorimetric glass transition temperature $T_{\mathrm{g}}$ (Ref. 13) and thereafter progressively reheated to $300 \mathrm{~K}$, while isothermal $( \pm 0.2 \mathrm{~K})$ frequency scans were performed at every fifth degree in the frequency range $10^{-2}-10^{7} \mathrm{~Hz}$. The imaginary part of the dielectric function was then fitted by one conductivity term and three Cole-Cole (CC) functions ${ }^{14}$ [Eq. (1)]:

$$
\begin{aligned}
\varepsilon^{*}(\omega)= & \varepsilon^{\prime}(\omega)-i \varepsilon^{\prime \prime}(\omega)=-i\left(\frac{\sigma_{0}}{\varepsilon_{0} \omega}\right)^{N} \\
& +\sum_{k=1}^{n}\left(\frac{\Delta \varepsilon_{k}}{1+\left(i \omega \tau_{k}\right)^{\alpha_{k}}}\right)+\varepsilon_{\infty k},
\end{aligned}
$$

where $\omega=2 \pi f$ is the angular frequency, $\varepsilon_{0}$ is the permittivity of free space, and $\sigma_{0}$ is the specific dc-conductivity. For each process/peak $k$ the relaxation time is given by $\tau$ and the dielectric strength by $\Delta \varepsilon$. $\alpha$ is the shape parameter that describes the symmetric broadening of the loss peak and $\varepsilon_{\infty}$ gives the value of the real part of the permittivity $\left(\varepsilon^{\prime}\right)$ at an infinite frequency. It should here be noted that, in contrast to many other supercooled liquids, also the $\alpha$-relaxation is symmetric and thereby fitted by the $\mathrm{CC}$ equation. ${ }^{15}$

\section{RESULTS AND DISCUSSION}

In Fig. 1 we show dielectric loss spectra of the three investigated monoalcohols, both in bulk and confinement. As obvious from the figure, the Debye-like process can be observed in all systems, although it becomes somewhat faster in the clay. Thus, it is even observed in the quasi-onedimensional geometry of the molecular sieve, which therefore excludes all possible structural models that are based on twodimensional or three-dimensional cluster formations as the origin of the Debye-like process. Also the $\alpha$-relaxation is observed in all systems, as more expected, and as the Debye-like process it becomes slightly faster in the confinement between clay platelets. This directly evident information is, of course, an important key for understanding the structural origin of the Debye-like process, but for a more detailed investigation of the confinement induced alterations of the Debye-like process we need to fit the dielectric loss data with Eq. (1) as explained above and shown in Fig. 1(a). From this curve fitting we obtained values of the fitting parameters given in Eq. (1) as a function of temperature. For instance, it becomes clear that the shape of the Debye-like peak becomes broader when the liquid is confined. In bulk the shape parameter $\alpha$ is $0.99-1.0$ for all the three monoalcohols, whereas in clay the values are $0.91(2 \mathrm{E} 1 \mathrm{H}), 0.85$ (2E1B), and 0.88 (2M1B), respectively. For $2 \mathrm{E} 1 \mathrm{H}$ confined in the pores of the molecular sieve the shape parameter varies from 0.77 (low T) to 0.91 (high T).

Values of the relaxation times are shown in the Arrhenius plot of Fig. 2. These $\tau$-values confirm the speeding up of the Debye-like process in the clay and show that in 2E1H, 2E1B, and $2 \mathrm{M} 1 \mathrm{~B}$ it becomes roughly a factor $3,3.5$, and 4.7 faster in the clay, compared to bulk. The corresponding speeding up of the $\alpha$-relaxation is $1.5(2 \mathrm{E} 1 \mathrm{H}), 2$ (2E1B), and 4.7 (2M1B), respectively. For $2 \mathrm{E} 1 \mathrm{H}$ confined in the molecular
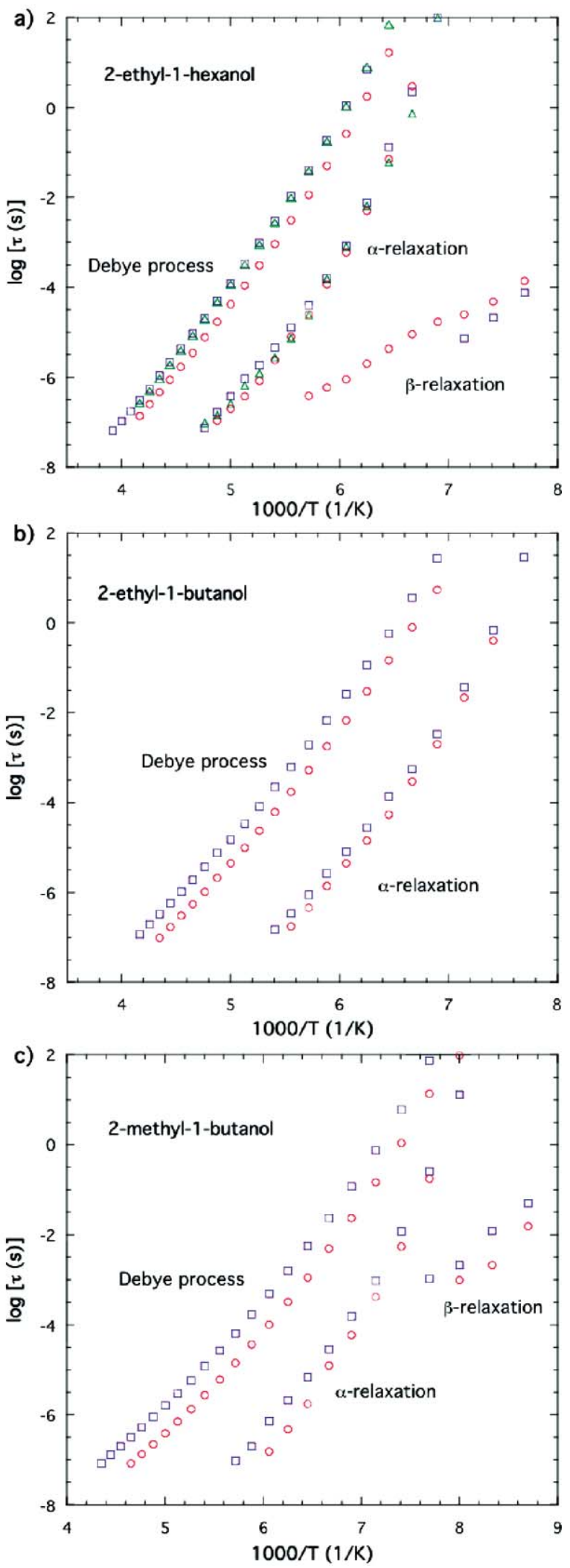

FIG. 2. Dielectric relaxation times obtained from the curve fitting shown in Fig. 1; (a) 2-ethyl-1-hexanol, (b) 2-ethyl-1-butanol, and (c) 2-methyl-1butanol. Relaxation times for bulk, clay, and molecular sieve samples are given by the symbols $\square, \mathrm{O}$, and $\Delta$, respectively. 
sieve the relaxation times for the $\alpha$-relaxation is somewhat faster in confinement than in bulk, but approximately the same for the Debye-like relaxation. The reason for why both the $\alpha$-relaxation and the Debye-like process become faster in the clay is likely the confinement induced speeding up, as discussed above. However, it should also be noted that the geometrical confinements are likely causing the densities of the confined liquids to be slightly lower compared to the corresponding bulk liquids, particularly in the case of the molecular sieve where a close packing of the molecules is not possible. The smaller change of the relaxation times in the molecular sieve may then be caused by competing surface effects.

It is also interesting to compare the relative dielectric strengths of the $\alpha$-relaxation and the Debye-like process. The ratio of the relative dielectric strengths between confinement and bulk can be defined as ${ }^{16}$

$$
\frac{\left.\frac{\Delta \varepsilon_{\alpha}}{\Delta \varepsilon_{\alpha}+\Delta \varepsilon_{D}}\right|_{\text {confined }}}{\left.\frac{\Delta \varepsilon_{\alpha}}{\Delta \varepsilon_{\alpha}+\Delta \varepsilon_{D}}\right|_{\text {bulk }}} \text { and } \frac{\left.\frac{\Delta \varepsilon_{D}}{\Delta \varepsilon_{\alpha}+\Delta \varepsilon_{D}}\right|_{\text {confined }}}{\left.\frac{\Delta \varepsilon_{D}}{\Delta \varepsilon_{\alpha}+\Delta \varepsilon_{D}}\right|_{\text {bulk }}}
$$

for the $\alpha$-relaxation and the Debye-like process, respectively. In Fig. 3 these ratios are shown as a function of temperature. The results indicate that all three monoalcohols behave in a similar way in the clay confinement, although the temperature dependence of the $\alpha$-relaxation might be somewhat different for $2 \mathrm{E} 1 \mathrm{H}$ compared to the other monoalcohols. If we compare the $\alpha$-relaxation of $2 \mathrm{E} 1 \mathrm{H}$ in clay and molecular sieve we observe, however, similar behavior. The relative dielectric strength of the Debye-like process is almost temperature independent and identical for the three monoalcohols, as well as for the two different types of confinement. By comparing the relative dielectric strength of the $\alpha$-relaxation with that of the Debye-like process it is clear that the dielectric strength of the Debye-like process decreases much more in confinement than the dielectric strength of the $\alpha$-relaxation. Even less affected by the confinement is the $\beta$-relaxation (not shown), which implies that the dielectric relaxation strength in confinement decreases more and more, compared to bulk, with an increasing time-scale of the relaxation process.

The overall finding that the Debye-like process is present in the quasi-one-dimensional pores of the molecular sieve implies that the only possibility of having larger structural formations of hydrogen bonded alcohol molecules is one-dimensional chainlike structures. If we assume that such a chainlike structure of hydrogen bonded alcohol molecules is formed in monoalcohols it is interesting to make a comparison with polymers. It is well known that some polymers exhibit an ultraslow (i.e., slower than the viscosity related $\alpha$-relaxation) process that reflects the overall chain dynamics. This slow relaxation is called the normal mode and since it is related to the motion of the entire chains (fluctuations of the end-to-end vector) its time-scale
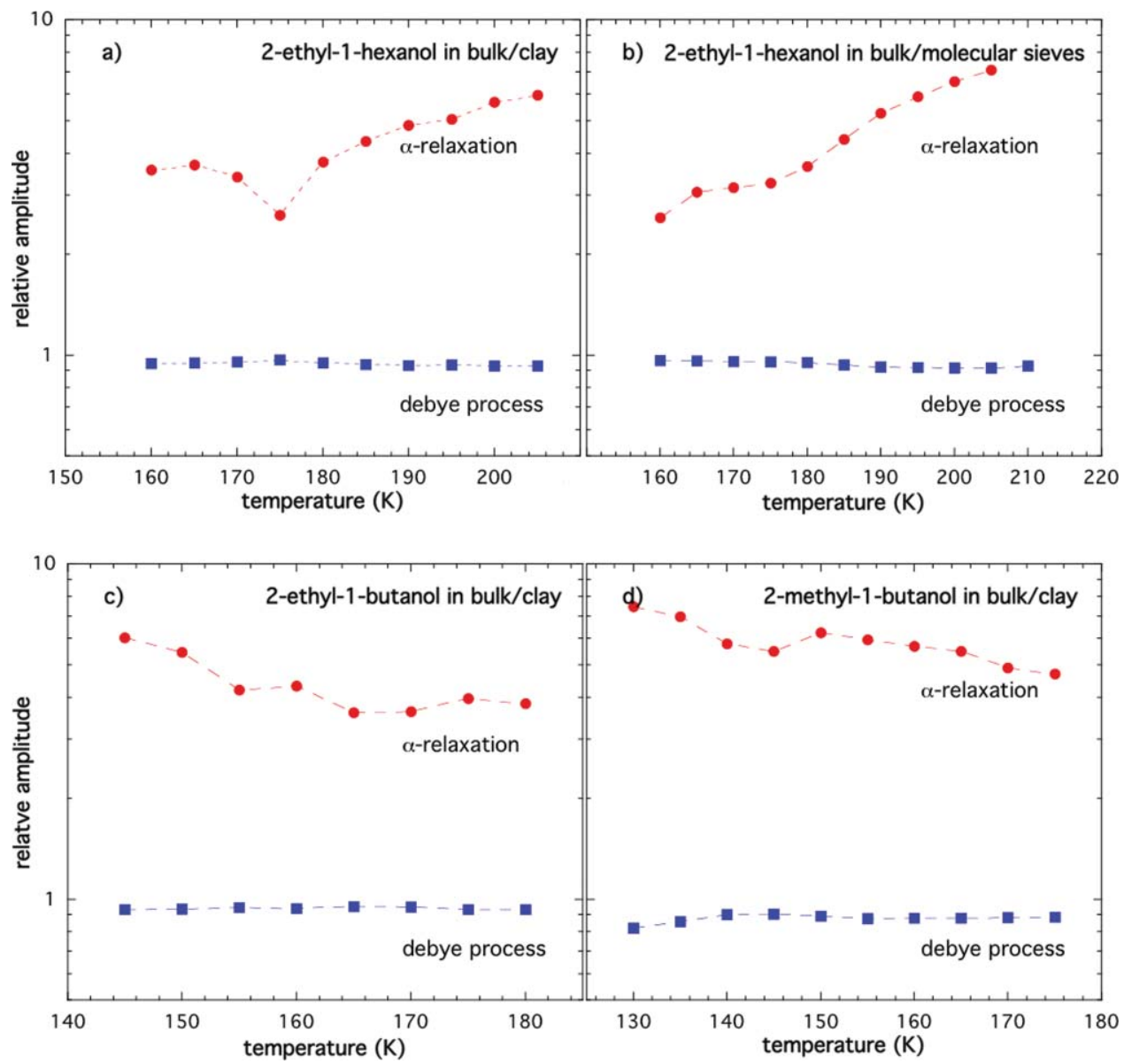

FIG. 3. Ratios of relative dielectric strengths between confinement and bulk [see Eq. (2)] for (a) 2-ethyl-1-hexanol in clay/bulk, (b) 2-ethyl-1-hexanol in molecular sieve/bulk, (c) 2-ethyl-1-butanol in clay/bulk, and (d) 2-methyl-1-butanol in clay/bulk. 
is strongly dependent on the molecular weight, i.e., the size, of the polymer chains. The behavior of this normal mode in the confinement of the same Na-vermiculite clay as used in this study has previously been investigated for different molecular weights of poly(propylene glycol) (PPG) by dielectric spectroscopy. ${ }^{12,16,17}$ From those studies it was found that the relaxation times of the normal mode process $\left(\tau_{\mathrm{NM}}\right)$ were significantly slowed down in the confinement compared to the relaxation times in the bulk polymers. In contrast, the time-scale of the $\alpha$-relaxation $\left(\tau_{\alpha}\right)$ was almost unaffected by the confinement. Furthermore, it was found that the relative dielectric strength of the normal mode was larger in confinement than in bulk, ${ }^{16}$ in contrast to the $\alpha$-relaxation where an opposite behavior was observed. Similar findings have been obtained from dielectric studies of PPG confined in vycor glasses of different pore sizes, ${ }^{18}$ although the $\alpha$-relaxation was generally speeded up at low temperatures in these confinements. Thus, both the slowing down and the increased relative dielectric strength of the normal mode in confinements are inconsistent with the findings for the Debye-like process in the confined monoalcohols. Therefore, a more probable explanation for the speeding up and decrease in the relative dielectric strength of the Debye-like process in the clay is that this process has a different origin than the normal mode in polymers. Another reason for that the normal mode analogy probably is incorrect is that the chain length of hydrogen bonded alcohol molecules is not expected to be well defined. Rather, the chains are expected to rapidly break up and reform in different ways, giving a wide distribution of chain lengths at a given time. This implies that the Debye-like process is expected to be considerably broader than the Debye shape if it is caused by the same physical mechanism as the normal mode. Hence, the almost perfect Debye shape of the Debye-like process in monoalcohols suggests that it is caused by a different kind of motion than the normal mode, although the monoalcohols may still form chain structures to some extent. Due to the small alteration of the time-scale of the Debye-like process in confinements it seems likely that the responsible motion is of local character and possibly related to the lifetime or breaking and reformation of single hydrogen bonds. In favor of this interpretation is also the observation that the intensity of the Debye-like process decreases more than the intensity of the $\alpha$-relaxation in the confinements, since it is likely that both the reduced density and the possibility of forming hydrogen bonds to the surfaces ${ }^{19}$ should reduce the average number of hydrogen bonds between the molecules.

\section{CONCLUSIONS}

Surprisingly, we have discovered that the slow dielectric Debye-like relaxation in monoalcohols is almost preserved in the quasi-two-dimensional space between clay platelets and in the quasi-one-dimensional pores of a molecular sieve. Thus, by restricting the monoalcohol molecules into these severe confinements, where extended network structures are impossible, we are able to exclude structural models that are based on two-dimensional or three-dimensional cluster formations as the origin of their Debye-like process. One-dimensional chain-like structures of hydrogen bonded alcohol molecules can be formed, but the present results show that it is unlikely that the Debye-like process is related to the motion of such chains, in analogy to the normal mode process in polymers. Instead, the results suggest that the Debye-like process is of more local character and related to the short-range interaction between the alcohol molecules.

\section{ACKNOWLEDGMENTS}

This work was financially supported by the Swedish Research Council and the Swedish Energy Agency.

${ }^{1}$ C. Hansen, F. Stickel, T. Berger, R. Richert, and E. W. Fischer, J. Chem. Phys. 107, 1086 (1997); L. M. Wang and R. Richert, ibid. 121, 11170 (2004).

${ }^{2}$ T. El Goresy and R. Bohmer, J. Chem. Phys. 128, 154520/1 (2008).

${ }^{3}$ B. Jakobsen, C. Maggi, T. Christensen, and J. C. Dyre, J. Chem. Phys. 129, 184502 (2008).

${ }^{4}$ Y. Yomogida, A. Minoguchi, and R. Nozaki, Phys. Rev. E 73, 041510 (2006); R. Bergman, H. Jansson, and J. Swenson, J. Chem. Phys. 132, 044504 (2010); Phys. Rev. Lett. 104, 017802 (2010).

${ }^{5}$ K. Kaminski, E. Kaminska, P. Wlodarczyk, K. Adrjanowicz, Z. Wojnarowska, K. Grzybowska, and M. Paluch, J. Phys.: Condens. Matter 22, 365103 (2010)

${ }^{6}$ A. R. Bras, J. P. Noronha, A. M. M. Antunes, M. M. Cardoso, A. Schonhals, F. Affouard, M. Dionisio, and N. T. Correia, J. Phys. Chem. B 112, 11087 (2008).

${ }^{7}$ H. Huth, L. M. Wang, C. Schick, and R. Richert, J. Chem. Phys. 126, 104503 (2007).

${ }^{8}$ A. K. Sum and S. I. Sandler, J. Phys. Chem. A 104, 1121 (2000); S. W. Benson, J. Am. Chem. Soc. 118, 10645 (1996); A. K. Karmakar, S. Sarkar, and R. N. Joarder, J. Phys. Chem. 99, 16501 (1995).

${ }^{9}$ R. G. Wassink and P. Bordewijk, Adv. Mol. Relax. Interact. Processes 13, 299 (1978).

${ }^{10}$ F. Affouard and N. T. Correia, J. Phys. Chem. B 114, 11397 (2010).

${ }^{11}$ N. T. Skipper, A. K. Soper, and J. D. C. McConnell, J. Chem. Phys. 94, 5751 (1991).

${ }^{12}$ G. A. Schwartz, R. Bergman, and J. Swenson, J. Chem. Phys. 120, 5736 (2004).

${ }^{13}$ L. M. Wang, Y. J. Tian, R. P. Liu, and R. Richert, J. Chem. Phys. 128, 084503 (2008).

${ }^{14}$ K. S. Cole and R. H. Cole, J. Chem. Phys. 9, 341 (1941).

${ }^{15}$ C. Gainaru and R. Bohmer, J. Non-Cryst. Solids 356, 542 (2010).

${ }^{16}$ G. A. Schwartz, R. Bergman, J. Mattsson, and J. Swenson, Eur. Phys. J. E 12, S113 (2003).

${ }^{17}$ J. Swenson, G. A. Schwartz, R. Bergman, and W. S. Howells, Eur. Phys. J. E 12, 179 (2003).

${ }^{18}$ A. Schonhals, H. Goering, and C. Schick, J. Non-Cryst. Solids 305, 140 (2002).

${ }^{19}$ A. R. Bras, S. Frunza, L. Guerreiro, I. M. Fonseca, A. Corma, L. Frunza, M. Dionisio, and A. Schonhals, J. Chem. Phys. 132, 224508 (2010). 